Pacific Journal of Mathematics

XPANSIVE AUTOMORPHISMS OF BANACH SPACES 


\section{EXPANSIVE AUTOMORPHISMS OF BANACH SPACES}

\section{Murray Eisenberg aNd JAMES H. HedLUND}

This paper treats two classes of invertible bounded linear operators on Banach spaces-expansive and uniformly expansive automorphisms-which include the hyperbolic automorphisms. Conditions for an automorphism to be expansive or uniformly expansive are given in terms of the location of its spectrum and approximate point spectrum with respect to the unit circle.

One of the tools used in [3] to determine all expansive automorphisms of compact connected Lie groups was the following result:

THEOREM 0. Let $T$ be an automorphism of a finite-dimensional real or complex normed linear space. Then a necessary and sufficient condition for $T$ to be expansive is that $|\lambda| \neq 1$ for each complex characteristic root $\lambda$ of $T$.

Theorem 0 was deduced in [2] as a special case of a more general theorem, concerning topological vector spaces over arbitrary nondiscrete scalar fields, whose proof used algebraic methods leaning heavily on the assumption of finite dimensionality. In the present paper we use analytic considerations to treat the infinite-dimensional case.

The results we obtain were suggested by the following observation. In the finite-dimensional case, the condition for an automorphism to be expansive amounts to its being hyperbolic for some norm; in any Banach space, an automorphism is hyperbolic for some norm precisely when its spectrum is disjoint from the unit circle.

1. Preliminaries. If $B$ is a real or complex Banach space, we shall call any bounded linear operator on $B$ having a bounded inverse on $B$ an automorphism of $B$.

The most convenient definition of "expansive" for our purposes is the following.

Definition 1. An automorphism $T$ of a Banach space $B$ is said to be expansive provided for each $x \in B$ with $\|x\|=1$ there exists some nonzero integer $i$ such that $\left\|T^{i} x\right\| \geqq 2$.

In this definition any norm equivalent to the given norm on $B$ may be used, and the constant 2 may be replaced by any constant 
strictly greater than 1. Moreover, an automorphism $T$ of $B$ is expansive if and only if there exists a constant $c>0$ such that $0 \neq x \in B$ implies $\left\|T^{i} x\right\| \geqq c$ for some integer $i$. Hence $T$ is expansive precisely when it is "unstable," in the sense of Utz [9], for the norm metric, and precisely when it is "expansive," in the sense of Bryant [1], for the uniformity which $B$ possesses as an abelian topological group.

The property named next will turn out to be something new only in the infinite-dimensional case.

Definition 2. An automorphism $T$ of a Banach space $B$ is said to be uniformly expansive provided there exists some positive integer $i$ such that $x \in B$ with $\|x\|=1$ implies $\left\|T^{i} x\right\| \geqq 2$ or $\left\|T^{-i} x\right\| \geqq 2$.

Here again, any equivalent norm may be used, and 2 may be replaced by any constant $c>1$.

We recall the following definition which will play an auxiliary role.

Definition 3. An automorphism $T$ of a Banach space $B$ is said to be hyperbolic provided there is a splitting

$$
B=B_{s} \oplus B_{u}, \quad T=T_{s} \oplus T_{u},
$$

where $B_{s}$ and $B_{u}$ are closed $T$-invariant linear subspaces of $B, T_{s}=T \mid B_{s}$ is a proper contraction (that is, $\left\|T_{s}\right\|<1$ ), and $T_{u}=T \mid B_{u}$ is a proper dilation (that is, $\left\|T_{u}^{-1}\right\|<1$ ).

For an automorphism $T$, we denote its spectrum by $\Lambda(T)$, its compression spectrum by $\Gamma(T)$, its approximate point spectrum by $\Pi(T)$, its point spectrum by $\Pi_{0}(T)$, and its spectral radius by $r(T)$. We denote the unit circle $\{\lambda:|\lambda|=1\}$ in the complex plane by $C$.

The lemma below is well known (compare [6]), but a proof is included for the sake of completeness.

Lemma 1. Let $T$ be an automorphism of a complex Banach space $B$. Then $T$ is hyperbolic with respect to some norm equivalent to the given norm of $B$ if and only if $\Lambda(T) \cap C=\varnothing$.

Proof. Assume that for some equivalent norm $\|\cdot\|$ we have a splitting $B=B_{s} \oplus B_{u}, T=T_{s} \oplus T_{u}$ as in Definition 3. Since $\left\|T_{s}\right\|<1$ and $\left\|T_{u}^{-1}\right\|<1, \Lambda\left(T_{s}\right)$ and $\Lambda\left(T_{u}\right)=\left(\Lambda\left(T_{u}^{-1}\right)\right)^{-1}$ are both disjoint from $C$, so the same is true of $\Lambda(T)$.

Conversely, assume $\Lambda(T) \cap C=\varnothing$. Define 


$$
\begin{aligned}
& \Lambda_{s}=\{\lambda \in \Lambda(T):|\lambda|<1\}, \\
& \Lambda_{u}=\{\lambda \in \Lambda(T):|\lambda|>1\} .
\end{aligned}
$$

By the spectral decomposition theorem (see, for example, [8, §148]), there exist closed $T$-invariant linear subspaces $B_{s}$ and $B_{u}$ of $B$ such that $B=B_{s} \oplus B_{u}, T=T_{s} \oplus T_{u}, \Lambda\left(T_{s}\right)=\Lambda_{s}$, and $\Lambda\left(T_{u}\right)=\Lambda_{u}$, where $T_{s}$ and $T_{u}$ are the restrictions of $T$ to $B_{s}$ and $B_{u}$ respectively. Since $r\left(T_{s}\right)<1$ and $r\left(T_{u}^{-1}\right)<1$, we may renorm $B_{s}$ and $B_{u}$ so that $\left\|T_{s}\right\|<1$ and $\left\|T_{u}^{-1}\right\|<1$ (see, for example, [5]). Then these norms on $B_{s}, B_{u}$ may be used to renorm $B$, and $T$ is hyperbolic for this new norm.

\section{Main results.}

THEOREM 1. Let $T$ be an automorphism of a complex Banach space $B$. Then:

(1) If $\Lambda(T) \cap C=\varnothing$, then $T$ is uniformly expansive.

(2) If $T$ is uniformly expansive, then $\Pi(T) \cap C=\varnothing$.

(3) If $\Pi(T) \cap C=\varnothing$, then $T$ is expansive.

(4) If $T$ is expansive, then $\Pi_{0}(T) \cap C=\varnothing$.

(5) The converses of implications (1), (3), and (4) all fail, even when $B$ is a Hilbert space.

Proof. (1) In view of Lemma 1, we need only show that $T$ is uniformly expansive provided it is hyperbolic for the given norm of $B$. Assume we have a $T$-invariant splitting $B=B_{s} \oplus B_{u}, T=T_{s} \oplus T_{u}$ with $\left\|T_{s}\right\|<1,\left\|T_{u}^{-1}\right\|<1$. Choose a constant $c$ with

$$
\max \left\{r\left(T_{s}\right), r\left(T_{u}^{-1}\right)\right\}<c<1 .
$$

By the spectral radius formula, there exists a positive integer $i$ such that

$$
\left\|T_{s}^{i}\right\|<c^{i}, \quad\left\|T_{u}^{-i}\right\|<c^{i}, \quad c^{i} \leqq 1 / 4 .
$$

Renorm $B$ by setting

$$
|x|=\left\|x_{s}\right\|+\left\|x_{u}\right\|
$$

when $x=x_{s}+x_{u}$ with $x_{s} \in B_{s}, x_{u} \in B_{u}$.

We show $T$ is uniformly expansive using this new norm. Let $x \in B$ with $|x|=1$. Write $x=x_{s}+x_{u}$, where $x_{s} \in B_{s}, x_{u} \in B_{u}$. Then $\left\|x_{s}\right\| \geqq 1 / 2$ or $\left\|x_{u}\right\| \geqq 1 / 2$. If $\left\|x_{s}\right\| \geqq 1 / 2$, then $\left\|x_{s}\right\| \leqq\left\|T_{s}^{i}\right\| \cdot\left\|T_{s}^{-i} x_{s}\right\|$, so

$$
\left|T^{-i} x\right| \geqq\left\|T_{s}^{-i} x_{s}\right\| \geqq\left\|T_{s}^{i}\right\|^{-1}\left\|x_{s}\right\| \geqq 4(1 / 2)=2 .
$$

Similarly, if $\left\|x_{u}\right\| \geqq 1 / 2$, then $\left|T^{i} x\right| \geqq 2$.

(2) Suppose there exists some $\lambda \in \Pi(T) \cap C$. Since the properties 
that $T$ be uniformly expansive and that $\Pi(T)$ be disjoint from $C$ are unaffected when $T$ is multiplied by a scalar of modulus 1 , we may assume $\lambda=1$. There exists a sequence $\left(x_{n} \mid n=1,2, \cdots\right)$ in $B$ with $\left\|x_{n}\right\|=1$ for each $n$ and $\left\|T x_{n}-x_{n}\right\| \rightarrow 0$. Then $\left\|x_{n}-T^{i} x_{n}\right\| \rightarrow 0$ for every integer $i$. For small $\varepsilon>0$ and any integer $i$, there is some $n$ for which $\left\|T^{i} x_{n}\right\|<1+\varepsilon$ and $\left\|T^{-i} x_{n}\right\|<1+\varepsilon$. Hence $T$ is not uniformly expansive.

(3) Suppose $T$ is not expansive; in other words, suppose there exists $x \in B$ with $\|x\|=1$ and $\left\|T^{n} x\right\| \leqq 2$ for every integer $n$. Define $E$ to be the least closed linear subspace of $B$ which is invariant under $T$ and $T^{-1}$ and which contains $x$, and set $S=T \mid E$. Clearly $S$ is not expansive. Since $\Pi(S) \subset \Pi(T)$, it suffices to show $\Pi(S) \cap C \neq \varnothing$.

We show first that $|\lambda| \leqq 1$ for each $\lambda \in \Gamma(S)$. Let $\lambda \in \Gamma(S)$. Then $\lambda \in \Pi_{0}\left(S^{*}\right)$, where $S^{*}$ is the automorphism induced by $S$ on the dual $E^{*}$ of $E$. Choose $y^{*} \in E^{*}$ with $\left\|y^{*}\right\|=1$ and $S^{*} y^{*}=\lambda y^{*}$. By definition of $E,\left\langle S^{i} x, y^{*}\right\rangle \neq 0$ for some integer $i$; set $c=\left\langle S^{i} x, y^{*}\right\rangle$. For each positive integer $n$,

$$
\left\langle S^{i+n} x, y^{*}\right\rangle=\left\langle S^{i} x,\left(S^{*}\right)^{n} y^{*}\right\rangle=\left\langle S^{i} x, \lambda^{n} y^{*}\right\rangle=\lambda^{n} c,
$$

so that

$$
2 \geqq\left\|S^{i+n} x\right\| \geqq\left|\left\langle S^{i+n} x, y^{*}\right\rangle\right|=|\lambda|^{n}|c| \text {. }
$$

It follows that $|\lambda| \leqq 1$. (Actually, $\Gamma(S) \subset C$. In fact, the same argument as above but with negative $n$ shows that $|\lambda| \geqq 1$ for each $\lambda \in \Gamma(S)$.)

We have $A(S) \cap C \neq \varnothing$, for otherwise $S$ would be (uniformly) expansive by (1). Choose $\lambda \in \Lambda(S) \cap C$. Either $\lambda \in$ bdy $\Lambda(S)$ or $\lambda \in \operatorname{int} \Lambda(S)$. If $\lambda \in \operatorname{bdy} \Lambda(S)$ we are done, since bdy $\Lambda(S) \subset \Pi(S)$. Suppose $\lambda \in \operatorname{int} \Lambda(S)$. Choose a sequence $\left(\lambda_{n} \mid n=1,2, \cdots\right)$ in $\Lambda(S)$ such that $\left|\lambda_{n}\right|>1$ for each $n$ and $\lambda_{n} \rightarrow \lambda$. By what was proved above, no $\lambda_{n} \in \Gamma(S)$, hence each $\lambda_{n} \in \Pi(S)$. Since $\Pi(S)$ is closed, $\lambda \in \Pi(S)$ also.

The proof of (4) is trivial and valid for any normed space. The examples in the next section establish (5).

COROLLARY 1. Each hyperbolic automorphism of $B$ is uniformly expansive.

Corollary 2. When $B$ is finite-dimensional, an automorphism of $B$ is expansive if and only if it is uniformly expansive.

Although we are unable to determine whether, in general, an automorphism is uniformly expansive if its approximate point spectrum is disjoint from $C$, we do have the following special result. 
THEOREM 2. Let $T$ be an automorphism of a complex Hilbert space $H$. Then a necessary and sufficient condition for $T$ to be uniformly expansive is that $\Pi(T) \cap C=\varnothing$.

For the proof we require an algebraic lemma.

LeMma 2. Given any $n$ complex numbers $c_{1}, \cdots, c_{n}$, there exists $\lambda \in C$ such that $\operatorname{Re}\left(\sum_{j=1}^{n} \lambda^{j} c_{j}\right) \geqq 0$.

Proof. Define $f$ by $f(z)=\sum_{j} c_{j} z^{j}$, let $\Gamma=f(C)$, and suppose $0 \notin \Gamma$. It will suffice to show that the winding number Ind $[\Gamma, 0]$ of $\Gamma$ with respect to 0 is positive, for then $\arg f(\lambda)=0$ for some $\lambda \in C$. We have

$$
\text { Ind }[\Gamma, 0]=\frac{1}{2 \pi i} \int_{\Gamma} \frac{d z}{z}=\frac{1}{2 \pi i} \int_{C} \frac{f^{\prime}(\lambda) d \lambda}{f(\lambda)} .
$$

Now $f$ is analytic inside $C$ and $f(0)=0$, so by the argument principle Ind $[\Gamma, 0] \geqq 1$.

Proof of Theorem 2. Necessity follows from Theorem 1. To prove sufficiency, suppose $T$ is not uniformly expansive. Then for each positive integer $n$ there exists $x_{n} \in H$ with $\left\|x_{n}\right\|=1$ and

$$
\max \left\{\left\|T^{n} x_{n}\right\|,\left\|T^{-n} x_{n}\right\|\right\}<2 .
$$

We are going to use Lemma 2 to construct for each $n$ a vector $y_{n} \in H$ and a number $\lambda_{n} \in C$ such that $\left\|\left(T-\lambda_{n} I\right) y_{n}\right\| \leqq A(\log n)^{-1 / 2}\left\|y_{n}\right\|$, where $A$ is a fixed constant independent of $n$. The desired conclusion will then follow. In fact, let $\lambda \in C$ be a limit point of $\left\{\lambda_{n}: n=1,2, \cdots\right\}$. Given $\varepsilon>0$,

$$
\begin{aligned}
\left\|(T-\lambda I) y_{n}\right\| & \leqq\left\|\left(T-\lambda_{n} I\right) y_{n}\right\|+\left\|\left(\lambda_{n}-\lambda\right) y_{n}\right\| \\
& \leqq\left[A(\log n)^{-1 / 2}+\left|\lambda_{n}-\lambda\right|\right]\left\|y_{n}\right\| \\
& \leqq \varepsilon\left\|y_{n}\right\|
\end{aligned}
$$

for large enough $n$. Hence $\lambda \in \Pi(T)$.

Fix a positive integer $n$; we construct the desired $y_{n} \in H$ and $\lambda_{n} \in C$. Set

$$
y_{n}=\sum_{j=k}^{m-1} \lambda^{j} T^{j} x_{n},
$$

where $m \geqq 0$ and $k \leqq 0, k<m$, are integers still to be determined, depending only on $n$, and where $\lambda \in C$ is still to be determined, depending on $m$ and $k$. An easy computation gives

$$
\left\|y_{n}\right\|^{2}=\sum_{j=k}^{m-1}\left\|T^{j} x_{n}\right\|^{2}+2 \operatorname{Re} \sum_{j=1}^{m-1-k} \lambda^{j} c_{j}
$$


where each $c_{j}$ is a sum of terms of the form $\left(T^{i} x_{n}, T^{m-i} x_{n}\right)$. By Lemma 2 we may choose $\lambda \in C$ such that the second term above is nonnegative. Then

$$
\left\|y_{n}\right\|^{2} \geqq \sum_{j=k}^{m-1}\left\|T^{j} x_{n}\right\|^{2}
$$

Set $\lambda_{n}=\lambda^{-1}$. Then

$$
\left\|\left(T-\lambda_{n} I\right) y_{n}\right\| \leqq\left\|T^{m} x_{n}\right\|+\left\|T^{k} x_{n}\right\| .
$$

To determine $m$ and $k$, upon which the choice of $\lambda$ depends, we consider three cases.

Case (i). $\quad\left\|T^{j} x_{n}\right\|^{2} \geqq(\log n) / n$ for $0 \leqq j \leqq n$. Take $m=n$ and $k=0$. From $(*),\left\|y_{n}\right\|^{2} \geqq \log n$; from $(* *)$ and the choice of $x_{n}$,

$$
\left\|\left(T-\lambda_{n} I\right) y_{n}\right\| \leqq\left\|T^{n} x_{n}\right\|+\left\|x_{n}\right\| \leqq 3
$$

Hence

$$
\left\|\left(T-\lambda_{n} I\right) y_{n}\right\| \leqq 3(\log n)^{-1 / 2}\left\|y_{n}\right\|
$$

Case (ii). $\left\|T^{j} x_{n}\right\|^{2} \geqq(\log n) / n$ for $-n \leqq j \leqq 0$. Take $m=0$ and $k=-n$. Exactly as in case (i) we find

$$
\left\|\left(T-\lambda_{n} I\right) y_{n}\right\| \leqq 3(\log n)^{-1 / 2}\left\|y_{n}\right\| \text {. }
$$

Case (iii). Cases (i) and (ii) both fail to apply. Let

$$
c=\max \left\{\|T\|,\left\|T^{-1}\right\|\right\} ;
$$

$c \geqq 1$ since $T$ is not uniformly expansive. Then $\left\|T^{j} x_{n}\right\| \geqq c^{-j}$ and $\left\|T^{-j} x_{n}\right\| \geqq c^{-j}$ for every $j$. Since case (i) fails to hold there is an integer $m, 0<m \leqq n$, for which $\left\|T^{m} x_{n}\right\|^{2}<\log n / n$, and we take the least such $m$. Then $m$ satisfies $c^{-2 m} \leqq\left\|T^{m} x_{n}\right\|^{2}<\log n / n$, so that $m>B \log n$ with $B=(4 \log c)^{-1}$. Similarly, since case (ii) fails there is an integer $k,-n \leqq k<0$, with $\left\|T^{k} x_{n}\right\|^{2}<\log n / n$. We take the greatest such $k$, which must satisfy $k<-B \log n$.

By the choice of $m$ and $k,\left\|T^{j} x_{n}\right\| \geqq\left\|T^{k} x_{n}\right\|$ for all $k \leqq j<m$. From $(*)$ we obtain

$$
\left\|y_{n}\right\|^{2} \geqq(m-k)\left\|T^{k} x_{n}\right\|^{2} \geqq(2 B \log n)\left\|T^{k} x_{n}\right\|^{2} .
$$

Now

$$
\left\|T^{m} x_{n}\right\|^{2}<\log n / n \leqq\left\|T^{k+1} x_{n}\right\| \leqq c\left\|T^{k} x_{n}\right\| .
$$

From $(* *)$ we obtain $\left\|\left(T-\lambda_{n} I\right) y_{n}\right\| \leqq(1+c)\left\|T^{k} x_{n}\right\|^{2}$. Hence in this case

$$
\left\|\left(T-\lambda_{n} I\right) y_{n}\right\| \leqq(1+c)(2 B)^{-1 / 2}(\log n)^{-1 / 2}\left\|y_{n}\right\|
$$


To conclude the proof, set $A=\max \left\{3,(1+c)(2 \log c)^{1 / 2}\right\}$.

3. Examples. The examples below establish assertion (5) of Theorem 1.

EXAMPLE 1. The bilateral shift on separable Hilbert space is unitary and, a fortiori, not expansive, but it has no eigenvalues.

EXAMPLE 2. Let $H$ be a separable Hilbert space with orthonormal base $\left(e_{n} \mid n=1,2, \cdots\right)$ and let $T$ be the diagonal operator on $H$ given by $T\left(e_{n}\right)=\lambda_{n} e_{n}$, where $0 \neq \lambda_{n} \notin C$ and $\lambda_{n} \rightarrow 1$. Then $T$ is expansive, for $0 \neq x \in H$ implies $\left(x, e_{n}\right) \neq 0$ for some $n$, so that

$$
\|\left. T^{i} x\right|^{2} \geqq\left|\lambda_{n}\right|^{2 i}\left|\left(x, e_{n}\right)\right|^{2} \geqq 4
$$

for some integer $i$ sufficiently large in absolute value. However, $1 \in I I(T)$.

Notice that if we take $\lambda_{2}=\lambda_{1}$, the new automorphism $S$ of $H$ given by $S\left(e_{1}\right)=e_{1}$ and $S\left(e_{n}\right)=\lambda_{n} e_{n}$ for $n>1$ is not expansive, yet $\Lambda(S)=\Lambda(T)$.

The preceding is a counterexample to the converse of Theorem 1 (3) when $B$ is a Hilbert space. Next we construct a counterexample for an arbitrary separable Banach space.

ExAMPLe 3. Let $B$ be any (infinite-dimensional) separable Banach space. Choose a maximal biorthogonal family $\left(e_{n}, e_{n}^{*}\right)_{n}$ with $\left\|e_{n}^{*}\right\|=1$ for every index $n$. We may take the index class of this family to be $\{1,2, \cdots\}$, for it is countable since the unit sphere in the dual $B^{*}$ of $B$ is weak-* separable. The Hahn-Banach theorem says that $\left(e_{n}^{*} \mid n=1,2, \cdots\right)$ must be total. Let $K$ be the compact operator given by $K=\sum_{n} \lambda_{n}\left\langle\cdot, e_{n}^{*}\right\rangle e_{n}$, where $\lambda_{n}=3^{-n}\left\|e_{n}\right\|^{-1} \leqq 3^{-n}, n=1,2, \cdots$. Let $T=I+K$, where $I$ is the identity operator. Since $\|K x\| \leqq\|x\| / 2$, $\|T x\| \geqq\|x\| / 2$ for all $x \in B$. Hence $T$ is an automorphism of $B$. Clearly $1 \in \Pi(T)$.

To see that $T$ is expansive, let $x \in B$ with $\|x\|=1$. For some $n,\left\langle x, e_{n}^{*}\right\rangle \neq 0$. For each positive integer $i,\left\langle T^{i} x, e_{n}^{*}\right\rangle=\left(1+\lambda_{n}\right)^{i}\left\langle x, e_{n}^{*}\right\rangle$, so $\left\|T^{i} x\right\| \geqq\left(1+\lambda_{n}\right)^{2}\left|\left\langle x, e_{n}^{*}\right\rangle\right|$. Hence $\left\|T^{i} x\right\| \geqq 2$ for large enough $i$.

ExAmPLE 4. Let $H$ be a separable Hilbert space. We construct a uniformly expansive automorphism $T$ of $H$ with

$$
\Lambda(T)=A=\{\lambda: 1 /(2 \sqrt{2}) \leqq|\lambda| \leqq 2 \sqrt{2}\} .
$$


More specifically, $\Pi_{0}(T)=\varnothing, \Pi(T)=$ bdy $A$, and $C \subset \operatorname{int} A=\Gamma(T)=$ $\Pi_{0}\left(T^{*}\right)$, so that the adjoint $T^{*}$ of $T$ is not even expansive.

Let $\left(e_{n}\right)_{-\infty}^{\infty}$ be an orthonormal base of $H$. Define the weighted bilateral shift $T$ of $H$ by

$$
T e_{n}=\left\{\begin{array}{l}
2 \sqrt{2} e_{n+1}, n \geqq 0, \\
\frac{1}{2 \sqrt{2}} e_{n+1}, n \leqq-1
\end{array}\right.
$$

To see that $T$ is uniformly expansive, let $x \in H$ with $\|x\|=1$. Write $x=y+c e_{0}+z$, where $y$ is orthogonal to $e_{n}$ for all $n \leqq 0$ and $z$ is orthogonal to $e_{n}$ for all $n \geqq 0$. Now $\|y\|^{2}+|c|^{2}+\|z\|^{2}=\|x\|^{2}=1$, If $\|y\|^{2}+|c|^{2} \geqq 1 / 2$, then

$$
\|T x\|^{2} \geqq(2 \sqrt{2})^{2}\left(\|y\|^{2}+|c|^{2}\right) \geqq 4 ;
$$

if $|c|^{2}+\|z\|^{2} \geqq 1 / 2$, then $\left\|T^{-1} x\right\|^{2} \geqq(2 \sqrt{2})^{2}\left(|c|^{2}+\|z\|^{2}\right) \geqq 4$.

To determine $\Lambda(T)$, note initially that $\left\|T^{n}\right\|=\left\|T^{-n}\right\|=(2 \sqrt{2})^{n}$ for every $n \geqq 1$. Hence $\Lambda(T) \subset A$. Now $T^{*}$ is the weighted backwards shift

$$
T^{*} e_{n}=\left\{\begin{array}{l}
2 \sqrt{2} e_{n-1}, n \geqq 1, \\
\frac{1}{2 \sqrt{2}} e_{n-1}, n \leqq 0
\end{array}\right.
$$

To find $\Pi_{0}\left(T^{*}\right)$, fix a complex number $\lambda \neq 0$. Then $T^{*} x=\lambda x$ for some vector $0 \neq x=\sum_{-\infty}^{\infty} c_{n} e_{n}$ if and only if

$$
\lambda c_{n}=\left\{\begin{array}{l}
\frac{1}{2 \sqrt{2}} c_{n+1}, n \leqq-1, \\
2 \sqrt{2} c_{n+1}, n \geqq 0,
\end{array},\right.
$$

that is, $c_{n}=(\lambda / 2 \sqrt{2})^{n} c_{0}$ and $c_{-n}=(1 / 2 \sqrt{2} \lambda)^{n} c_{0}$ for all $n \geqq 1$. Since $x \neq 0$, the series $\sum c_{n} e_{n}$ converges if and only if $|\lambda / 2 \sqrt{2}|<1$ and $|1 / 2 \sqrt{2} \lambda|<1$, that is, $\lambda \in \operatorname{int} A$.

It follows that $\Lambda(T) \supset \operatorname{cls} \Gamma(T)=A$. A computation similar to the above shows that $I_{0}(T)=\varnothing$, and elementary estimates give that $I I(T)=\operatorname{bdy} A$.

Example 5. Let $A$ be the annular region $\{z: 1 / 2<|z|<2\}$ in the complex plane and let $H$ be the space of all analytic functions belonging to the complex Hilbert space $L^{2}(A, m)$, where $m$ is planar Lebesgue measure. Then [4] $H$ is a separable Hilbert space. The analytic position operator $T$ on $H$ given by $T f(z)=z f(z)$ is an auto- 
morphism of $H$. It is proved in [4] that $\Lambda(T)$ is the closure of $A$ and $I(T)$ is the boundary of $A$. Thus $C \subset \Lambda(T)$, yet $T$ is uniformly expansive by Theorem 2 .

We sketch a direct proof that $T$ is uniformly expansive which avoids the computation of $\Lambda(T)$ given in [4]. Take $f \in H$ with $\|f\|=1$ and define

$$
\varphi(r)=r \int_{0}^{2 \pi}\left|f\left(r e^{i t}\right)\right|^{2} d t \quad(1 / 2<r<2) .
$$

Thus $1=\|f\|^{2}=\int_{1 / 2}^{2} \varphi(r) d r$. Since $f$ is analytic, $\varphi(r)>0$. It follows from Hölder's inequality that $\log \varphi(r)$ is a convex function of $\log r$ : if $r=r_{1}^{t} r_{2}^{1-t}$ with $0 \leqq t \leqq 1$, then $\varphi(r) \leqq \varphi\left(r_{1}\right)^{t} \varphi\left(r_{2}\right)^{1-t}$. One concludes that $\varphi$ is a continuous function having no local maximum in $(1 / 2,2)$. Since $\int_{1 / 2}^{2} \varphi(r) d r=1$, either $\int_{1}^{2} \varphi(r) d r \geqq 2 / 3$ or $\int_{1 / 2}^{1} \varphi(r) d r \geqq 1 / 3$. In the former case the geometrical properties of $\varphi$ imply that $\int_{3 / 2}^{2} \varphi(r) d r \geqq 1 / 3$, so that

$$
\begin{aligned}
\left\|T^{n} f\right\|^{2} & =\int_{1 / 2}^{2} r^{2 n} \varphi(r) d r \\
& \geqq(3 / 2)^{2 n} \int_{3 / 2}^{2} \varphi(r) d r \geqq(3 / 2)^{2 n}(1 / 3)
\end{aligned}
$$

for all $n \geqq 1$. Similarly, in the latter case $\int_{1 / 2}^{3 / 4} \varphi(r) d r \geqq 1 / 6$, so that $\left\|T^{-n} f\right\|^{2} \geqq(4 / 3)^{2 n} / 6$. Hence $\max \left\{\left\|T^{n} f\right\|,\left\|T^{-n} f\right\|\right\} \geqq(4 / 3)^{n} / \sqrt{6}$ for all $n \geqq 1$.

4. Remarks. Implications (1) and (3) of Theorem 1 no longer hold if we take real instead of complex scalars. In fact, let $B^{*}$ be any nonzero complex Banach space and let $T^{*}$ be the multiplication in $B^{*}$ by the complex number $i$. Then the automorphism $T$ of the real Banach space subordinate to $B^{*}$ is an isometry, but $\Lambda(T)=\varnothing$.

Now let $T$ be any automorphism of a real Banach space $B$. Denote by $T^{*}$ the induced automorphism of the complexification $B^{*}$ of $B$ (see [7, Th. 1.3.1], for example). There is a norm-preserving real-linear isomorphism $f$ of $B$ into $B^{*}$ with $f T=T^{*} f$; also $B^{*}=B \oplus B$ as a real Banach space, and $T^{*}=T \oplus T$. Hence $T$ is hyperbolic (uniformly expansive, expansive, respectively) precisely when $T^{*}$ is. It follows that Theorem 1 (1)-(4) remain valid in the real case if $\Lambda(T), \Pi(T), \Pi_{0}(T)$ are replaced by $\Lambda\left(T^{*}\right), \Pi\left(T^{*}\right), \Pi_{0}\left(T^{*}\right)$.

We return to the complex case. The set of all uniformly expansive automorphisms of a Banach space $B$ is easily seen to be open in the 
set of all automorphisms of $B$. It would be interesting to know whether the former set is dense in the latter.

Added in proof. Techniques similar to those used here show that Theorem 2 remains valid for an arbitrary Banach space. Also, the uniformly expansive automorphisms are not even dense in the expansive automorphisms. Proofs will appear in a paper by the secondnamed author.

\section{REFERENCES}

1. B. F. Bryant, On expansive homeomorphisms, Pacific J. Math. 10 (1960), 1163-1167. 2. M. Eisenberg, Expansive automorphisms of finite dimensional vector spaces, Fund. Math. 59 (1966), 307-312.

3. - Expansive transformation semigroups of endomorphisms, Fund. Math. 59 (1966), 313-321.

4. P. R. Halmos, G. Lumer, and J. J. Schäffer, Square roots of operators, Proc. Amer. Math. Soc. 4 (1953), 142-149.

5. R. B. Holmes, A formula for the spectral radius of an operator, Amer. Math. Monthly 75 (1968), 163-166.

6. C. C. Pugh, On a theorem of P. Hartman, Amer. J. Math. 91 (1969), 363-367.

7. C. E. Rickart, General theory of Banach alqebras, Van Nostrand, Princeton, 1960.

8. F. Riesz and B. Sz.-Nagy, Functional analysis, Ungar, New York, 1955.

9. W. R. Utz, Unstable homeomorphisms, Proc. Amer. Math. Soc. 1 (1950), 769-774.

Received February 7, 1970. The work of the first-named author was supported in part by National Science Foundation Grants GP-6651 and GP-11662.

University of Massachusetts, Amherst 


\section{PACIFIC JOURNAL OF MATHEMATICS}

\section{EDITORS}

H. SAMELSON

Stanford University

Stanford, California 94305

\section{Richard Pierce}

University of Washington

Seattle, Washington 98105
J. DugundJI

Department of Mathematics

University of Southern California

Los Angeles, California 90007

RICHARD ARENS

University of California

Los Angeles, California 90024

\section{ASSOCIATE EDITORS}

\section{E. F. BeCKenBACH}

B. H. NeUmanN
K. YosHida

\section{SUPPORTING INSTITUTIONS}

\author{
UNIVERSITY OF BRITISH COLUMBIA \\ CALIFORNIA INSTITUTE OF TECHNOLOGY \\ UNIVERSITY OF CALIFORNIA \\ MONTANA STATE UNIVERSITY \\ UNIVERSITY OF NEVADA \\ NEW MEXICO STATE UNIVERSITY \\ OREGON STATE UNIVERSITY \\ UNIVERSITY OF OREGON \\ OSAKA UNIVERSITY \\ UNIVERSITY OF SOUTHERN CALIFORNIA
}

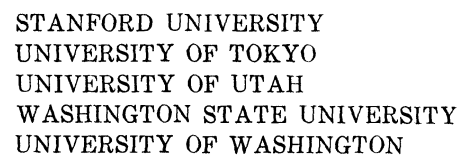

STANFORD UNIVERSITY UNIVERSITY OF TOKYO

UNIVERSITY OF UTAH

WASHINGTON STATE UNIVERSITY

UNIVERSITY OF WASHINGTON

The Supporting Institutions listed above contribute to the cost of publication of this Journal, but they are not owners or publishers and have no responsibility for its content or policies.

Mathematical papers intended for publication in the Pacific Journal of Mathematics should be in typed form or offset-reproduced, (not dittoed), double spaced with large margins. Underline Greek letters in red, German in green, and script in blue. The first paragraph or two must be capable of being used separately as a synopsis of the entire paper. The editorial "we" must not be used in the synopsis, and items of the bibliography should not be cited there unless absolutely necessary, in which case they must be identified by author and Journal, rather than by item number. Manuscripts, in duplicate if possible, may be sent to any one of the four editors. Please classify according to the scheme of Math. Rev. Index to Vol. 39. All other communications to the editors should be addressed to the managing editor, Richard Arens, University of California, Los Angeles, California, 90024.

50 reprints are provided free for each article; additional copies may be obtained at cost in multiples of 50 .

The Pacific Journal of Mathematics is published monthly. Effective with Volume 16 the price per volume (3 numbers) is $\$ 8.00$; single issues, $\$ 3.00$. Special price for current issues to individual faculty members of supporting institutions and to individual members of the American Mathematical Society: $\$ 4.00$ per volume; single issues $\$ 1.50$. Back numbers are available.

Subscriptions, orders for back numbers, and changes of address should be sent to Pacific Journal of Mathematics, 103 Highland Boulevard, Berkeley, California, 94708.

PUBLISHED BY PACIFIC JOURNAL OF MATHEMATICS, A NON-PROFIT CORPORATION

Printed at Kokusai Bunken Insatsusha (International Academic Printing Co., Ltd.), 7-17, Fujimi 2-chome, Chiyoda-ku, Tokyo, Japan. 


\section{Pacific Journal of Mathematics}

\section{Vol. 34, No. 3 \\ July, 1970}

Richard Hindman Bouldin, The peturbation of the singular spectrum

Hugh D. Brunk and Søren Glud Johansen, A generalized Radon-Nikodym derivative .

Henry Werner Davis, F. J. Murray and J. K. Weber, Families of $L_{p}$-spaces

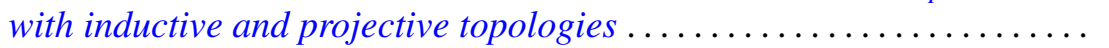

Esmond Ernest Devun, Special semigroups on the two-cell .

Murray Eisenberg and James Howard Hedlund, Expansive automorphisms

of Banach spaces ......................................

Frances F. Gulick, Actions of functions in Banach algebras.

Douglas Harris, Regular-closed spaces and proximities.

Norman Lloyd Johnson, Derivable semi-translation planes . .

Donald E. Knuth, Permutations, matrices, and generalized Young

tableaux..........................................

Herbert Frederick Kreimer, Jr., On the Galois theory of separable

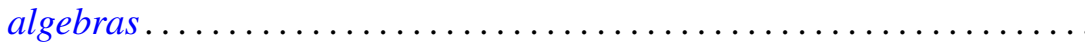

You-Feng Lin and David Alon Rose, Ascoli's theorem for spaces of

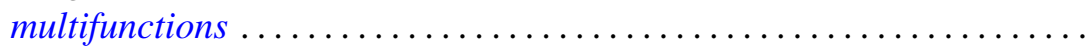

David London, Rearrangement inequalities involving convex functions . . . .

Louis Pigno, A multiplier theorem.

749

Helga Schirmer, Coincidences and fixed points of multifunctions into trees.

755

Richard A. Scoville, Some measure algebras on the integers .

Ralph Edwin Showalter, Local regularity of solutions of Sobolev-Galpern

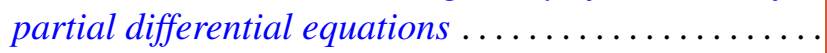

Allan John Sieradski, Twisted self-homotopy equivalences

John H. Smith, On S-units almost generated by S-units of subfields ...

803

Masamichi Takesaki, Algebraic equivalence of locally normal

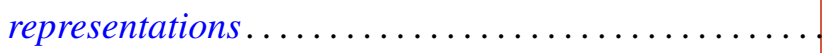

Joseph Earl Valentine, An analogue of Ptolemy's theorem and its converse in

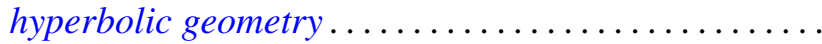

David Lawrence Winter, Solvability of certain p-solvable linear groups of finite order 